\title{
Dealing With Conflicts of Interest
}

Journals such as International Journal of Sports Physiology and Performance (IJSPP) are generally accepted as trusted sources of information by researchers and practitioners. This position of influence that research journals hold in these professions is becoming increasingly important in the information age where new (often not peer-reviewed) information is more readily available through the Internet and social media. The trust in journals as valued, reliable information sources has been built on the independent peer-review process where their content is evaluated by independent experts. However, there are circumstances where the public trust can be put at risk. A conflict of interest (COI) from an author, a reviewer, or an editor can influence the trustworthiness of a paper. In this editorial, I examine the potential sources of COI in sport-related research and discuss how they affect the perceived trustworthiness of research. I also examine how COIs might occur and the influence of these COIs, and then provide some directions for how authors and reviewers should respond to a COI.

A COI can be described as a circumstance-or set of circumstances - where professional interests are excessively influenced by an individual's secondary interest(s). ${ }^{1}$ In the context of scientific publishing, a COI is a situation where an individual (ie, an author, reviewer, or editor) who is in a position of trust has a competing professional or personal interest such that a reasonable observer might wonder if the individual's judgment or behavior might be motivated by his/her competing interests. Such competing interests can make it difficult to fulfill one's duties impartially, but they do not necessarily lead to unethical or improper actions. Nonetheless, COIs are of significant concern for researchers as they may either advertently or inadvertently influence the integrity of research.

While the majority of researchers follow ethical practices, there are cases where some fail to disclose COIs that may undermine the trustworthiness of their work. In sport-related research, it appears that COIs are less commonly disclosed. This may be due to authors' lack of awareness of their responsibility to declare conflicts, or even a lack of awareness of a possible conflict. There are also specific considerations in sport-related research, such as the influence of in-kind support, personal status, or financial gain, that need to be carefully considered. Nonetheless, while the level of funding and external support for sport-performance research is often lower than heavily privately funded research areas (eg, biomedical or pharmaceuticals), similar COIs occur for sportrelated researchers. These may occur when

- A researcher is asked to review a paper that has been submitted by an author with whom she/he has a relationship (eg, collaborator, author from same institution, mentor, or former student).

- A journal editor or associate editor submits a paper to that journal.

- A researcher is asked to review a paper from a competing group.

- A researcher is asked to review a paper assessing the measurement precision of a device or the efficacy of a product from a company in which he/she holds shares or from which he/she receives financial reward.

- A researcher submits a paper that has findings that directly support his/her consulting business work.

An important consideration for researchers associated with projects that receive external financial support or provide direct personal benefit (regardless of their size) is that these types of incentives may influence the researcher's objective judgment and result in bias. Indeed, to gain further insight into these phenomena, it is valuable to understand how people respond to decisions with attached incentives. Studies have shown that incentives have an effect, with even small incentives having a powerful influence on researcher behavior. ${ }^{2,3}$ Accordingly, individuals who provide and/or accept gifts - even with an explicit "no strings attached" understanding - still carry an expectation of reciprocity. ${ }^{3}$ It has been suggested that the expectation of reciprocity may be the primary motive for providing small gifts or incentives (which are common in the pharmaceutical industry). ${ }^{4}$ On this basis, any level of support for a project or potential personal incentive related to a project should be carefully considered when declaring COIs.

\section{What Should You Do When You Have a COI?}

Having a COI does not mean that any impropriety has occurred. Indeed, each COI needs to be judged on its proportionality and the author's transparency. Like most journals, IJSPP has clear instructions to help contributors handle any potential COIs. Specifically, the journal requires that authors-for all submissions-identify any potential COIs, either financial, institutional, and/or personal relationships. Financial relationships that could form a potential COI include employment, consultancy, honoraria, and other payments. Personal COI can relate to personal relationships, academic or sporting competition, and intellectual passion. Authors must disclose potential conflicts of interest to the subjects in the study being reported and state this explicitly in the Methods section of their manuscripts. In instances where the study has been funded by a third party with a proprietary or financial interest in the outcomes, the corresponding author should declare these interests in the cover letter to the journal editor on submission and in the Acknowledgments section of the manuscript. By following these recommendations, authors have clear directions for dealing with COIs.

Although bias cannot be eliminated from our work, acknowledging potential COIs can help readers interpret the trustworthiness of our findings. A simple rule of thumb applies when dealing with $\mathrm{COI}$-we recommend that investigators play it safe. Indeed, we could move to follow more stringent approaches such as those of the International Committee of Medical Journal Editors, who ask contributors to disclose COIs by completing standard declarations (see http://www.icmje.org/conflicts-of-interest). Finally, readers should also accept that the presence of a $\mathrm{COI}$ is independent of the occurrence of impropriety. With this approach, sport scientists will set high 
standards in research integrity and trustworthiness for our work and help it continue to develop the field of sport-performance research.

Aaron J. Coutts, Associate Editor, IJSPP

\section{References}

1. Lo B, Field MJ. Conflict of Interest in Medical Research, Education and Practice. Washington, DC: National Academies Press; 2009.
2. Brennan TA, Rothman DJ, Blank L, et al. Health industry practices that create conflicts of interest: a policy proposal for academic medical centers. JAMA. 2006;295(4):429-433. PubMed ID: 16434633 doi:10. 1001/jama.295.4.429

3. Dana J, Loewenstein G. A social science perspective on gifts to physicians from industry. JAMA. 2003;290(2):252-255. PubMed ID: 12851281 doi:10.1001/jama.290.2.252

4. Cain DM, Loewenstein G, Moore DA. The dirt on coming clean: perverse effects of disclosing conflicts of interest. J Legal Stud. 2005; 34(1):1-25. doi:10.1086/426699 